\title{
Immunohistochemical detection of abnormal cell proliferation in colonic mucosa of subjects with polyps
}

\author{
R G Wilson, A N Smith, C C Bird
}

\begin{abstract}
Previous studies have shown the presence of increased proliferation in the large bowel epithelium of those at high risk of developing colon cancer. An in vitro technique for labelling large bowel mucosa with the thymidine analogue bromodeoxyuridine (Brdu) was therefore developed and its ability to distinguish differences in mucosal proliferation between subjects with colorectal adenomas and normal controls was assessed.
\end{abstract}

Sigmoid biopsy specimens from 15 subjects with polyps and 15 age and sex matched controls were labelled and the incorporated Brdu visualised with an immunohistochemical technique. Mean labelling index (LI) was significantly higher in those with polyps than in controls. Differences in the pattern of labelling in colonic crypts were compared by the generation of cumulative labelling distributions. Analysis showed a significant expansion of the proliferative compartment in the colon crypts of those with polyps.

It is concluded that in vitro labelling with Brdu provides a useful method for the assessment of mucosal proliferation in subjects at high risk of developing colon cancer.

Many studies have shown abnormalities of mucosal epithelial proliferation in diseases which predispose to gastrointestinal cancer. Autoradiography following ${ }^{3} \mathrm{H}$-Thymidine $\left({ }^{3} \mathrm{H}-\mathrm{T}\right)$ incorporation into mucosal fragments in vitro has been the most widely used technique for studying cell proliferation in subjects at increased risk for colonic neoplasia. ${ }^{1}$ Using this technique, significant differences in the labelling index (LI) and distribution of proliferative cells within large bowel crypts have been described in hereditary non-polyposis colon cancer, ${ }^{2}$ familial polyposis coli, ${ }^{34}$ and in the presence of sporadic polyps or carcinoma. ${ }^{56}$ In vitro ${ }^{3} \mathrm{H}$-T labelling has therefore been proposed as a potential method for identifying high risk subjects, ${ }^{75}$ and has also been used as an "intermediate biomarker" of colon cancer risk in assessing the short term effects of dietary intervention in man. ${ }^{89}$ It is an exacting technique, however, with the disadvantage of requiring the use of radioactive isotopes and considerable delays (often several weeks) while autoradiographs are developed.

The recent development of a monoclonal antibody against the thymidine analogue bromodeoxyuridine (Brdu) now permits much more rapid identification of S-phase cells (and thus the LI) by immunohistochemical techniques. ${ }^{10}$ Measurement of Brdu incorporation is comparable in sensitivity to that of ${ }^{3} \mathrm{H}-\mathrm{T}$ in vivo. ${ }^{11}$ We have therefore explored its use for in vitro labelling of human colorectal mucosa, and assessed its use as a means of discriminating those at increased risk of colon neoplasia by comparing its incorporation in the sigmoid mucosa from subjects with large or multiple colonic polyps and normal controls.

\section{Methods}

\section{LABELLING TECHNIQUE}

Biopsy specimens of sigmoid mucosa taken through a colonoscope were obtained from patients with one or more large adenomas $(>1$ $\mathrm{cm}$ ) and age and sex matched non-polyp bearing controls. All biopsy specimens from the patients were taken a minimum of $5 \mathrm{~cm}$ away from any macroscopic adenomas. Control subjects were selected on the basis of negative colonoscopy and barium enema in addition to having no family history of large bowel neoplasia. Biopsy specimens were taken between 9 and 11 am to exclude any theoretical effects of diurnal variation. ${ }^{12}$ Immeditely after removal specimens were transported to the laboratory in complete medium, pre-gassed with $95 \%$ oxygen and $5 \%$ carbon dioxide at $37^{\circ} \mathrm{C}$, and established in organ culture. The organ culture technique is essentially that described by Pritchett et al. ${ }^{13}$ Whole biopsy specimens were orientated, luminal surface

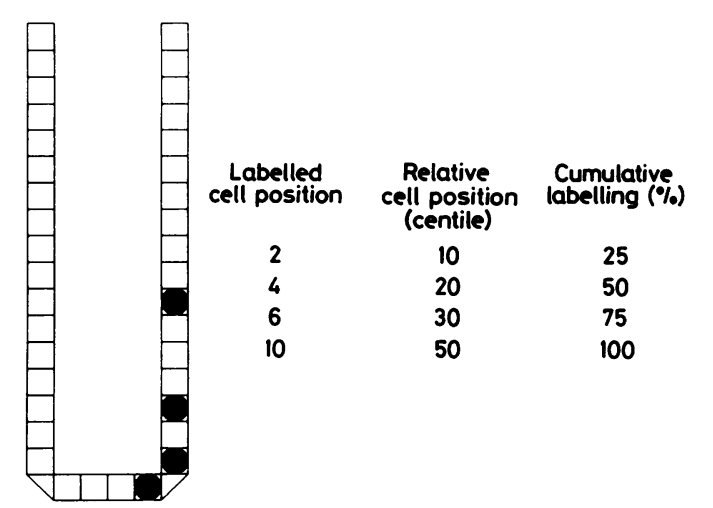

Figure 1 Stylised crypt column showing scoring of labelled cell positions and calculation of cumulative labelling distribution in a crypt containing 20 cells. 


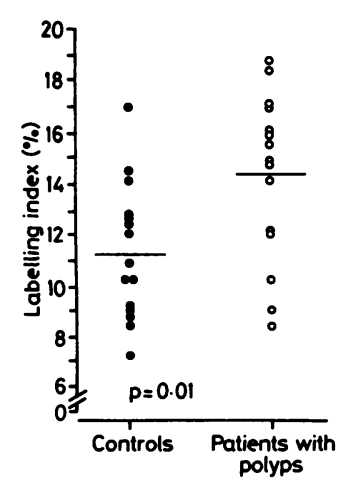

Figure 2 Labelling indices of controls and patients. $(-=$ mean LI). up, on steel grids and placed in organ culture dishes. The culture medium used comprised Weymouth's MB752 medium, supplemented with $10 \%$ fetal calf serum, L-glutamine 1.5 $\mathrm{mM}$, ascorbic acid $300 \mu \mathrm{g} / \mathrm{ml}$, hydrocortisone $3.0 \mu \mathrm{g} / \mathrm{ml}$, ferrous sulphate $0.45 \mu \mathrm{g} / \mathrm{ml}$ and gentamycin $50 \mu \mathrm{g} / \mathrm{ml},{ }^{14}$ to which $\mathrm{Brdu}$ was added to a final concentration of $100 \mu \mathrm{mol}$, and fluorodeoxyuridine at a concentration of $10 \mu$ mol. ${ }^{15}$ Tissue explants were maintained at the gas fluid interface in a sealed modular incubator containing $95 \%$ oxygen and 5\% carbon dioxide at a pressure of two atmospheres. Explants were labelled at $37^{\circ} \mathrm{C}$ for one hour, rinsed and fixed by submerging the grids in Carnoy's fixative for 12-16 hours.

The biopsy specimens were then processed to paraffin wax and carefully embedded on edge to ensure that subsequent sections were taken through the vertical crypt axis. Sections $3 \mu \mathrm{m}$ thick were cut at intervals of $100 \mu \mathrm{m}$ through each tissue block and plated onto poly L-lysine coated slides for subsequent immunostaining.

\section{IMMUNOSTAINING TECHNIQUE}

Sections were dewaxed and placed in $1^{\circ}{ }_{0}$ hydrogen peroxide $\left(\mathrm{H}_{2} \mathrm{O}_{2}\right)$ in methanol for 20 minutes to block endogenous peroxidase. After washing in TRIS buffered saline (TBS), $\mathrm{pH}$ $7 \cdot 6$, DNA was denatured by immersion of the slides in $1 \mathrm{~N} \mathrm{HCl}$ for eight minutes at $60^{\circ} \mathrm{C}^{16}$ to expose bound Brdu for immunostaining with an avidin-biotin peroxidase technique using a standard ABC kit (Dako). Tissue sections were incubated successively in $20^{\circ}$ or rabbit serum in TBS, $1 / 500$ mouse anti-Brdu (Becton-Dickinson) for 30 minutes at room temperature, 1/ 400 biotinylated rabbit anti-mouse for $30 \mathrm{~min}$ utes, and finally in peroxidase avidin-biotin complex for 30 minutes. After each step sections were rinsed in TBS. The antibody-peroxidase complex was visualised by incubation in $0.02 \mathrm{M}$ TRIS/ $\mathrm{HCl}$ buffer ( $\mathrm{pH} \mathrm{7.6)} \mathrm{containing}$ $0.05 \%$ diaminobenzidine and $0.1 \% \mathrm{H}_{2} \mathrm{O}_{2}$ for five minutes. Sections were counterstained with Meyer's haematoxylin, dehydrated through graded alcohol, cleared in Histoclear, and mounted in synthetic resin.

\section{SCORING OF BRDU LABELLING}

Stained sections were scored for Brdu labelling under a light microscope at a magnification of $\times 400$. Forty longitudinally sectioned hemicrypts (crypt-columns) were analysed from each patient. ${ }^{5}$ For each crypt column the total number of cells and the number and position of Brdu positive cells were directly keyed into a microcomputer for further analysis. The LI (percentage of cells in S-phase) for each patient was determined from the percentage of $\mathrm{Brdu}$ positive cells in each of the 40 crypt columns.

To discern differences in the distribution of labelled cells within crypts cumulative labelling distributions of Brdu positive cells were generated. This method entails normalising each crypt column to a notional 100 cell positions, with cell position 0 at the crypt base and cell position 100 at the luminal surface. The distribution of positive cells is then expressed as a cumulative percentage for each crypt cell position centile. For example, if a crypt column contains 20 cells and the second, fourth, sixth and tenth cells from the bottom are labelled then these cells are assigned to the 10th, 20th, 30 th, and 50 th centiles of that crypt. The cumulative labelling corresponding to these centiles would then be $25,50,75$ and $100 \%$ (fig 1). The results from all crypt columns in a sample can be combined to generate a cumulative labelling distribution for that sample.

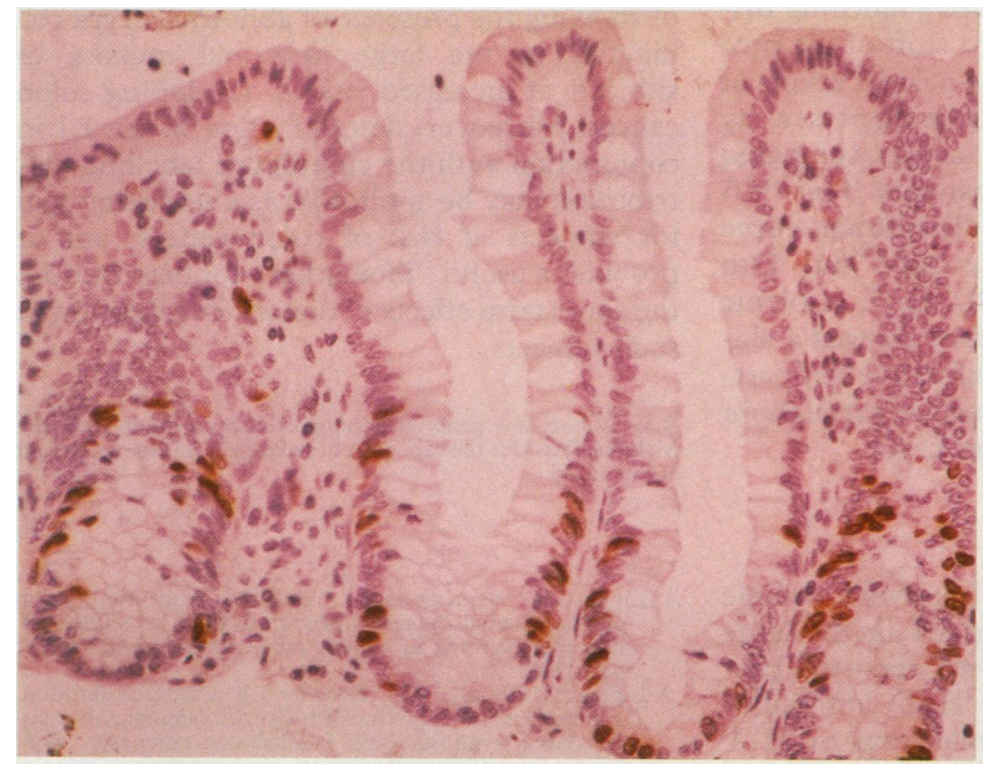

Figure 3 Photomicrograph of Brdu labelling from a control showing labelled cells confined to the basal region of the crypts.

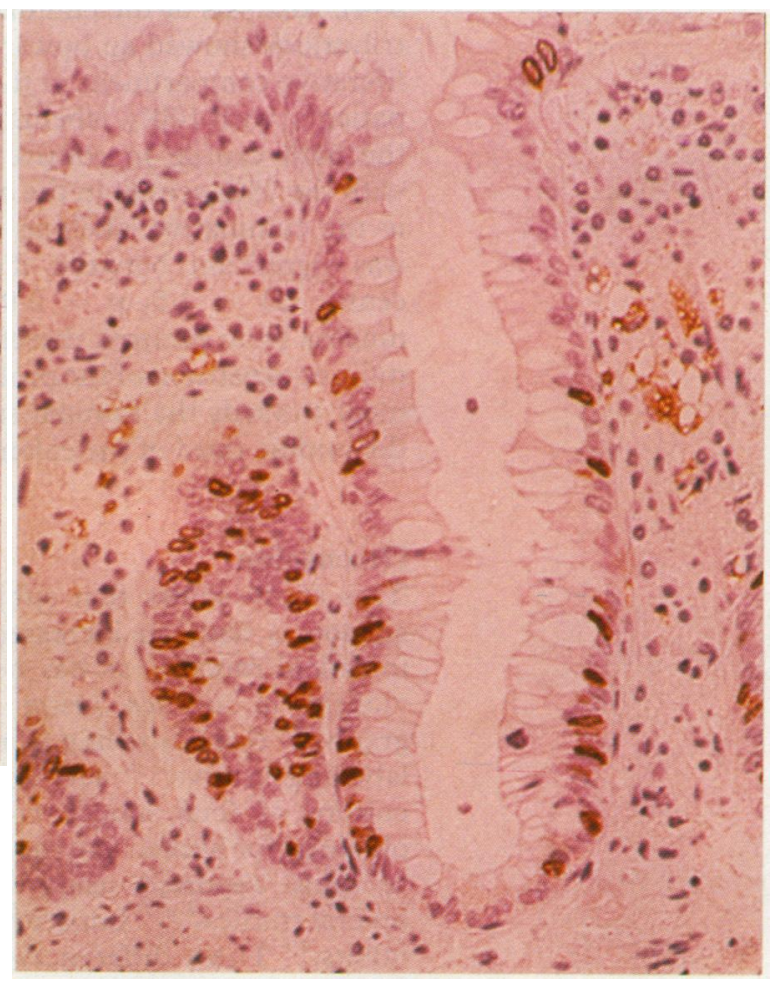


Figure 5 Cumulative labelling distributions in sigmoid mucosa of patients and controls. The curve is significantly shifted towards the right the luminal surface of the crypts) in polyp bearing mucosa.

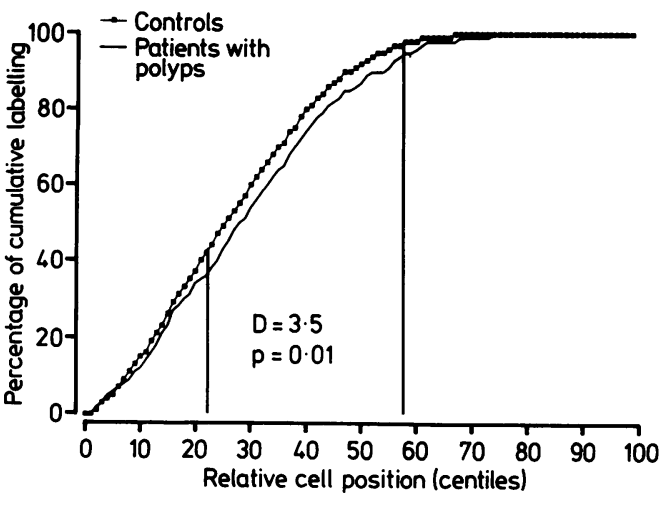

Comparison of LI and crypt column height were performed using Student's $t$ test. To test for significant differences between the cumulative labelling distribution of patients and control subjects a two sample two-tailed Kolmogorov-Smirnov analysis was used. This non-parametric test defines a critical value $D$ for any chosen probability at which the differences between cumulative distributions become significant. ${ }^{17}$

\section{Results}

Biopsy specimens were obtained from eight male and seven female patients with colonic polyps with a median age of 60 years (range 4179 ) and from 15 age and sex matched controls with a median age of 61 years (range 42-76).

Mean LI was significantly higher in the patients than in the control group (14.3 (SEM) $0.85 v 11.27(0.7), \mathrm{t}=2.76, \mathrm{p}=0.01)$, although there was considerable overlap in the range of values within the two groups (fig 2).

Mean crypt column size was similar in the controls and patients (56.6 (SD) $8.5 v 57.2$ $(8 \cdot 4), p=n s)$. In addition to differences in the LI, extension of the proliferative compartment was found in the mucosa from the patients (figs 3-4). Analysis of the cumulative labelling distributions showed an upward shift of the proliferative compartment in the colonic crypts of patients, with $100 \%$ labelling being present by the 64th centile in control crypts compared with the 75th centile in the patients. This shift in the cumulative labelling distribution curve was significant between the 22nd and 57th centile $(D=3.5, p=0.01$; fig 5$)$. Colonic mucosa from patients therefore showed a significant expansion of the proliferative compartment towards the luminal surface throughout the middle third of the colonic crypt.

\section{Discussion}

This study shows that subjects with colonic adenomas have an increased LI in the large intestinal mucosa, as has been reported previously in studies using ${ }^{3} \mathrm{H}-\mathrm{T}$ autoradiography. ${ }^{15}$ Brdu labelling is analogous to ${ }^{3} \mathrm{H}-\mathrm{T}$ labelling in vivo ${ }^{1118}$ and in vitro using organ culture of fetal mouse colon. ${ }^{19}$ The values obtained for human colonic mucosa are similar to those reported using ${ }^{3} \mathrm{H}-\mathrm{T}$ labelling in several studies, ${ }^{62021}$ but are higher than in some others. ${ }^{59}$ These differences may be explained by variations in the handling of biopsy material, the available amount of ${ }^{3} \mathrm{H}-\mathrm{T}$ in the culture medium, and the criteria used when scoring autoradiographs for labelled cells.

Risio et al reported a mean LI of $5 \cdot 7 \%$ using in vitro Brdu in 10 samples of normal descending colon, ${ }^{22}$ which contrasts with the mean value of $11.5 \%$ in this study. These authors counted fewer crypt columns, however, and experienced uneven or patchy uptake of Brdu. Our methods also differ from theirs in the use of an organ culture technique, the addition of fluorodeoxyuridine to enhance uptake of Brdu, ${ }^{15}$ and the presence of a hyperbaric gas phase during labelling. The presence of a hyperbaric gas phase produces better correlation between in vitro labelling immediately after biopsy and in vivo labelling with ${ }^{3} \mathrm{H}-\mathrm{T}^{23}$ and Brdu. ${ }^{11}$ Moreover, the LI values obtained with Brdu in this study are similar to those reported after intravenous infusion of Brdu in man. ${ }^{24}$

Traditionally, the distribution of the proliferative compartment within the colonic crypt has been analysed by dividing the crypt column into four, five, or even 10 separate compartments, with subsequent comparison of the LI within individual compartments. ${ }^{24-625}$ Analysis of autoradiographs (after ${ }^{3} \mathrm{H}-\mathrm{T}$ labelling) have shown an increase in proliferative activity in the upper portions of the colonic crypts in familial polyposis coli, ${ }^{27}$ hereditary non-polyposis colon cancer, ${ }^{2}$ and sporadic adenomas and carcinomas, ${ }^{56}$ compared with controls. The generation of cumulative labelling distributions shows a significant shift in the zone of major proliferative activity towards the luminal surface in the presence of sporadic adenomas in a manner analogous to the "stage 2 " proliferative defect described in previous studies. ${ }^{25} 26$

We conclude that in vitro labelling with Brdu is a rapid and sensitive method that is capable of showing the presence of abnormal crypt cell kinetics in the large intestinal mucosa of subjects at increased risk of developing colon. cancer. Moreover, determination of the LI in conjunction with the cumulative labelling distribution may be applicable as a screening tool for subjects at risk of colon cancer, and it provides a useful "biomarker" for determining the short term effects of dietary intervention in such subjects.

R G Wilson was generously supported by a grant from the Melville Trust for the care and cure of cancer during the period of this study.

1 Lipkin $M$. Biomarkers of increased susceptibility to gastrointestinal cancer: a new application to studies of cancer prevention in human subjects. Cancer Res 1988 48:235-45.

2 Lipkin M, Blattner WA, Gardner EJ, et al. Classification and risk assessment of individuals with familial polyposis Gardner's syndrome and familial non-polyposis colon cancer from $\left[{ }^{3} \mathrm{H}\right]$ thymidine labelling patterns in colonic epithelial cells. Cancer Res 1984;44:4201-7.

3 Iwana T, Utsunomiya J, Sasaki J. Epithelial cell kinetics in the crypts of familial polyposis colon. Jpn J Surg 1977;7: $230-4$.

4 Deschner EE, Lipkin M. Proliferative patterns in colonic mucosa in familial polyposis. Cancer 1975;35:413-18.

5 Terpstra OT, Van Blankenstein M, Dees J, Eilers GAM. Abnormal pattern of cell proliferation in the entire colonic 
mucosa of patients with colon adenoma or cancer. Gastroenterol 1987:92:704-48.

6 Ponz de Leon M, Roncucci L, DiDonato P, et al. Pattern of epithelial cell proliferation in colorectal mucosa of normal subjects and of patients with adenomatous polyps or cancer of the large bowel. Cancer Res 1988;48:4121-6.

7 Lipkin M. Method for binary classification and risk assessment of individuals with familial polyposis based on $\left[{ }^{3} \mathrm{H}\right]$ Tdr labelling of epithelial cells in colonic crypts. Cell Tissue Kinet 1984;17:209-22.

8 Lipkin M, Newark H. Effect of added dietary calcium on colonic epithelial cell proliferation in subjects at high risk colonic epithelial cell proliferation in subjects at high risk

9 Stadler J, Stern HS, Yeung KAS, et al. Effect of high fat consumption on cell proliferative activity of colorecta mucosa and on soluble faecal bile acids. Gut 1988; 29:1326-331.

10 Gratzner HG. Monoclonal antibody to 5-Bromo \& 5Iododeoxyuridine: a new reagent for detection of DNA replication. Science 1982;218:474-6.

11 Wilson GO, McNally NJ, Dunphy E, Kacher H, Pfaguer R The labelling index of human and mouse tumours assessed by bromodeoxyuridine staining in vitro and in vivo and flow cytometry. Cytometry 1985;6:641-7.

12 Al-Dewachi HS, Wright NA, Appleton DR, Watson AJ. Studies on the mechanism of diurnal variation of prodiferative indices in the small bowel mucosa of the rat. proliferative indices in the small
Cell Tissue Kinet 1976;9:459-67.

13 Pritchett CJ, Senior PV, Sunter JP, et al. Human colorectal tumours in short term organ culture. Cell Tissue Kinet 1982;15:555-64

14 Hodges GM, Melcher AH. Chemically defined medium for the growth and differentiation of mixed epithelial and connective tissue in organ culture. In-vitro 1976;12:450-6.

15 Ellwart J, Dormer P. Effect of 5-Fluoro 2-deoxyuridine (FdUrd) on 5-Bromo 2-deoxyuridine (BrdUrd) incorporation into DNA measured with a monoclonal BrdUrd and by the Brdu/Hoechst Quenching effect. Cytometry 1985;6:513-20.
16 Wynford-Thomas D, Williams ED. Use of bromodeoxyuridine for cell kinetic studies in intact animals. Cell Tissue Kinet 1986;19:179-82.

17 Siegel S. Nonparametric statistics for the behavioral sciences. (International student edition) Koga Kusha: McCrawHill, 1956:131-6.

18 Sughihara $H$, Hatton $T$, Fakuda $M$, Immunohistochemical detection of bromodeoxyuridine in formalin fixed tissues. Histochemistry 1986;85:193-5.

19 Morstyn G, Pyke K, Gardner J, Ashcroft R, deFazio A, Bathal P. Immunohistochemical identification of proliferating cells in organ culture using bromodeoxyuridine and a monoclonal antibody. J Histochem Cytochem 1986; and a monoclo

20 Kanemitsu T, Koike A, Yamamoto S. Study of the cell proliferation kinetics in ulcerative colitis, adenomatous polyps, and cancer. Cancer 1985;56:1094-8.

21 Bleiberg $H$, Buyse $M$, Galand $P$. Cell kinetic indicators of premalignant stages of colorectal cancer. Cancer 1985; 56:124-9.

22 Risio M, Courlizza S, Ferrari A, Candelaresi GL, Rossini FP. Immunohistochemical study of cell proliferation in hyperplastic polyps, adenomas and carcinomas of the large hyperplastic polyps, adenomas and carcind

23 Wargovitch MJ, Hu PJ. An improved method for determination of colonic mucosal proliferation: uptake of tritiated thymidine under hyperbaric conditions. Cancer Let 1988;42:207-12.

24 Khan S, Raza A, Petrelli N, Mittelman A. In vivo determination of labelling index of metastatic cancer and normal colonic mucosa using intravenous infusions of bromodeoxyuridine. $J$ Surg Oncol 1988;39:114-8.

25 Deschner EE, Maskens AP. Significance of the labelling index and labelling distribution as kinetic parameters in colorectal mucosa of cancer patients and DMH treated animals. Cancer 1982;50:1136-41.

26 Lipkin M. Phae 1 and phase 2 proliferative lesions of colonic epithelial cells in diseases leading to colon cancer. Cancer 1974;34:878-88. 